\title{
A automedicação da população mundial: uma revisão integrativa
}

\author{
The self-medication in the world population: an integrative review \\ La automedicación en la poblacion mundial: una revisión integrativa
}

\section{Resumo}

A automedicação é um fenômeno mundial e sua prevalência difere em função da população estudada, do método e do período recordatório utilizado. Sendo assim, a abordagem da temática se faz necessária vez que é uma questão de preocupação para as autoridades internacionais. Foi realizada pesquisa em bases de dados de Saúde, utilizando critérios de inclusão e seleção para seleção de artigos científicos. Então, foi observada a prática da automedicação em indivíduos com maior escolaridade. Ademais, em relação ao motivo da automedicação, foram encontrados relatos de experiência anterior no tratamento de sintomas recorrentes, a percepção de não haver gravidade nessa ação e não precisar de atendimento médico para tratamento dos sintomas relatados. As principais classes de medicamentos observados foram os analgésicos, anti-inflamatórios, antibióticos e fármacos com ação no sistema gastrointestinal. A automedicação é um fenômeno com possível nocividade e que deve ser compreendida e estudada de maneira aprofundada.

Palavras-chave: Automedicação; Uso de medicamentos; Efeitos adversos.

\begin{abstract}
Self-medication is a worldwide phenomenon and its prevalence differs according to the population studied, the method and the recall period used. Therefore, the approach to the theme is necessary since it is a matter of concern for international authorities. A research was carried out in health databases, using inclusion and selection criteria for the selection of scientific articles. Then, the practice of self-medication was observed in individuals with higher schooling. Moreover, regarding the reason for self-medication, reports of previous experience in the treatment of recurrent symptoms were found, the perception of no severity in this action and no need for medical attention to treat the reported symptoms. The main classes of drugs observed were analgesics, anti-inflammatory drugs, antibiotics and drugs with action on the gastrointestinal system. Self-medication is a phenomenon with possible harmfulness and that must be fully understood and studied.
\end{abstract}

Keywords: Self-medication; Medication use; Adverse effects. 


\begin{abstract}
Resumen
La automedicación es un fenómeno mundial y su prevalencia difiere según la población estudiada, el método y el período de recuerdo utilizado. Por tanto, el abordaje del tema es necesario ya que es un tema de preocupación para las autoridades internacionales. Se realizó una investigación en bases de datos en salud, utilizando criterios de inclusión y selección para la selección de artículos científicos. Luego, se observó la práctica de la automedicación en individuos con mayor escolaridad. Por otra parte, en cuanto al motivo de la automedicación, se encontraron relatos de experiencia previa en el tratamiento de síntomas recurrentes, la percepción de no gravedad en esta acción y no necesidad de atención médica para tratar los síntomas reportados. Las principales clases de fármacos observadas fueron analgésicos, antiinflamatorios, antibióticos y fármacos con acción sobre el sistema gastrointestinal. La automedicación es un fenómeno con posible nocividad y que debe entenderse y estudiarse plenamente.
\end{abstract}

Palabras clave: Automedicación; Uso de medicamentos; Efectos adversos.

\title{
1. Introdução
}

A automedicação é conceituada como a seleção e o uso de medicamentos sem prescrição ou supervisão de um médico ou dentista (Organização Mundial Da Saúde [OMS], 1998 citado em Arrais et al., 2016). Mesmo que muitas vezes esta prática possa ter resultados favoráveis, como a melhora de sintomas ou resolução do problema de saúde, outras vezes pode trazer prejuízos à saúde do indivíduo, mascarando os problemas de saúde, intoxicações, reações adversas, interações medicamentosas, desenvolvimento de resistência entre outros (Galato et al., 2012; Ogawa et al., 2001). Os fatores que contribuem para a sua efetivação podem estar relacionados à variedade de produtos fabricados pela indústria farmacêutica, pela facilidade de comercialização, bem como a questões culturalmente estabelecidas na sociedade contemporânea (Ministério da Saúde, 2012).

A automedicação é um fenômeno mundial e sua prevalência difere em função da população estudada, do método e do período recordatório utilizado (Arrais et al., 2016). Além disso, mais de 50\% de todos os medicamentos são incorretamente prescritos, dispensados, vendidos e, metade dos pacientes os utilizam de maneira errada (OMS, 2013 citado em Domingues et al., 2015). No Brasil, 79\% das pessoas com mais de 16 anos admitem tomar medicamentos sem prescrição médica ou farmacêutica (Instituto de Ciência, Tecnologia e Qualidade [ICTQ], 2018). 57\% dos entrevistados, especialmente homens (60\%) e jovens de 16 a 24 anos (69\%), passam pelo profissional de saúde, mas não usam o medicamento conforme orientado (Conselho Federal de Farmácia [CFF], 2019).

Entretanto, apesar de haver uma disparidade entre os métodos utilizados na pesquisa, devido à ausência de padronização metodológica em função da variação de faixa etária e sexo, além de suas diferenças socioeconômicas e culturais, a mesma conteve um inquérito que atendeu ao critério de participantes semelhantes, isso é, dentro do um mesmo contexto, possibilitando o trabalho (Mendes, 2010).

Diante disso, compreende-se que a problemática do tratamento medicamentoso por conta própria trouxe resultados importantes para a população, visto que cerca de um terço dos adultos o exerce. Como consequência disso, cabe citar: o agravamento de doenças; proliferação de microrganismos resistentes e, portanto, o comprometimento da eficácia de tratamentos; anulação ou potencialização do efeito do outro medicamento; reações alérgicas, intoxicação, dependência e, até mesmo, a morte (Ministério da Saúde, 2012). Ademais da automedicação constituir-se de uma prática permanente, a qual ainda pode ser estimulada por alguns meios, como a mídia televisiva e vários outros meios de comunicação e propaganda como o rádio ou "outdoors" insistem com seus apelos a estimular a população a adotar tal postura, inserindo no final da propaganda a frase "persistindo os sintomas um médico deve ser consultado", como se tal fato a isentasse de toda e qualquer responsabilidade" (Revista da Associação Médica Brasileira, 2001).

Sendo assim, a abordagem da temática em questão se faz necessária tendo em vista que os dados relativos à prevalência da automedicação no Brasil são escassos, mesmo sendo uma questão de preocupação para as autoridades governamentais. Logo, o fato de existir a dificuldade do acesso aos serviços de saúde pela população, a crença nos benefícios do tratamento/prevenção de doenças e a necessidade de aliviar sintomas devem ter prioridade no acervo de pesquisas para que sirva de estímulo ao uso 
racional de medicamentos pela população brasileira (OMS, 2004), podendo assim, ser reafirmado por Paulo e Zanine (1988), "a automedicação é um procedimento caracterizado fundamentalmente pela iniciativa de um doente, ou de seu responsável, em obter ou produzir e utilizar um produto que acredita lhe trará benefícios no tratamento de doenças ou alívio de sintomas.

Em suma, diante do exposto, é válido, então, reafirmar o objetivo central da pesquisa: realizar uma revisão da automedicação como uma problemática ainda existente em meio à realidade contemporânea. Nesse contexto, é necessário, também, salientar seus efeitos, bem como questões à ela associadas.

\section{Metodologia}

Trata-se de uma revisão integrativa de literatura, cuja finalidade é sintetizar resultados obtidos em pesquisas sobre um delimitado tema ou questão, de maneira sistemática e ordenada, com o objetivo de contribuir para o conhecimento desse tema ou questão (Roman \& Friedlander, 1998). Tem-se como base a seguinte pergunta norteadora: "Há nocividade para a população que pratica a automedicação?", que permitiu a análise e compilado dos trabalhos científicos disponíveis, os quais auxiliaram para uma prática baseada em evidências. Utilizou-se das 6 etapas da revisão integrativa: 1 - Identificação do tema e seleção da questão de pesquisa; 2 - Estabelecimento dos critérios de inclusão e exclusão; 3 - Identificação dos estudos pré-selecionados e selecionados; 4 - Categorização dos estudos selecionados; 5 - Análise e interpretação dos resultados; e 6 - Apresentação da revisão/síntese do conhecimento.

Foram buscados artigos, durante o mês de novembro de 2020, nas bases de dados SciELO (The Scientific Electronic Library Online), PubMed (United States National Library of Medicine) e LILACS (Literatura Latino-Americana e do Caribe em Ciências da Saúde), por meio de critérios de inclusão estabelecidos: publicação entre os anos de 2016 e 2020, artigos publicados nas línguas portuguesa e inglesa, com desenho de revisão sistemática. Além disso, também foram definidos critérios de exclusão: artigos que não tratassem de uma revisão sistemática e artigos incompletos. Já os critérios de elegibilidade foram trabalhos que não se relacionavam com o tema da pergunta norteadora e os que não estavam repetidos nas bases de dados. Foram usados os seguintes descritores indexados no sistema de Descritores em Ciências da Saúde (DeCS): automedicação, uso de medicamentos, efeitos adversos, comportamento e Brasil; além dos equivalentes na língua inglesa: self medication, drug utilization, adverse effects, behavior e Brazil. O operador booleano usado para combinar os descritores foi OR. Considerando as bases científicas analisadas, 10 referências atenderam aos critérios de seleção estabelecidos (Figura 1).

Em relação à análise metodológica, foi utilizado uma adaptação do Critical Appraisal Skill Programme (CASP): 1 Objetivo claro e justificado; 2 - Metodologia adequada; 3 - Apresentação e discussão dos procedimentos teóricos e metodológicos; 4 - Seleção adequada da amostra; 5 - Coleta de dados detalhada; 6 - Relação entre pesquisador e pesquisados; 7 - Aspectos éticos preservados; 8 - Análise de dados rigorosa e fundamentada; 9 - Apresentação e discussão dos resultados; 10 Contribuições, limitações e indicações de novas questões de pesquisa. De forma que, cada objetivo exposto acima poderá ter a pontuação de zero ou um, e cada trabalho tem uma pontuação máxima de 10 pontos, dependendo se ele foi identificado no artigo analisado. Dessa forma, os artigos selecionados foram divididos em duas classificações de qualidade de acordo com as suas pontuações: nível A (6 a 10 pontos) - trabalho de boa qualidade metodológica e viés reduzido; ou nível B (mínimo de 5 pontos) - trabalho com qualidade metodológica satisfatória, porém com risco de viés aumentado.

\section{Resultados e Discussão}

Dos 73.539 artigos achados, 64.303 não atenderam ao critério de inclusão "ano de publicação", 2.659 ao "idioma", 6.205 ao desenho de revisão sistemática, resultando em 372 artigos. Destes, 22 artigos atenderam ao critério de exclusão de artigos incompletos, restando 350. Destes, 340 não atenderam ao critério de elegibilidade "relação com o tema da pergunta" 
e não havia trabalhos repetidos. Resultando em 10 artigos na amostra final (Figura 1). As principais informações da amostra final encontram-se no Quadro 1. Em relação ao idioma, 8 artigos foram publicados em inglês, 02 em português. Dos anos de publicação, 01 artigo de 2016, seguido por 03 de 2017, 04 de 2018, 01 de 2019 e 01 de 2020. Os países dos estudos: 02 foram no Brasil, 02 da Etiópia, 01 da França, 01 da China, 01 da Índia, 01 do Nepal, 01 do Oriente médio, 01 dos países europeus e anglo-saxões. Posteriormente à leitura integral dos estudos, 09 artigos foram classificados como nível A e 01 como nível B, conforme CASP adaptado.

Figura 1. Fluxograma da amostra final.

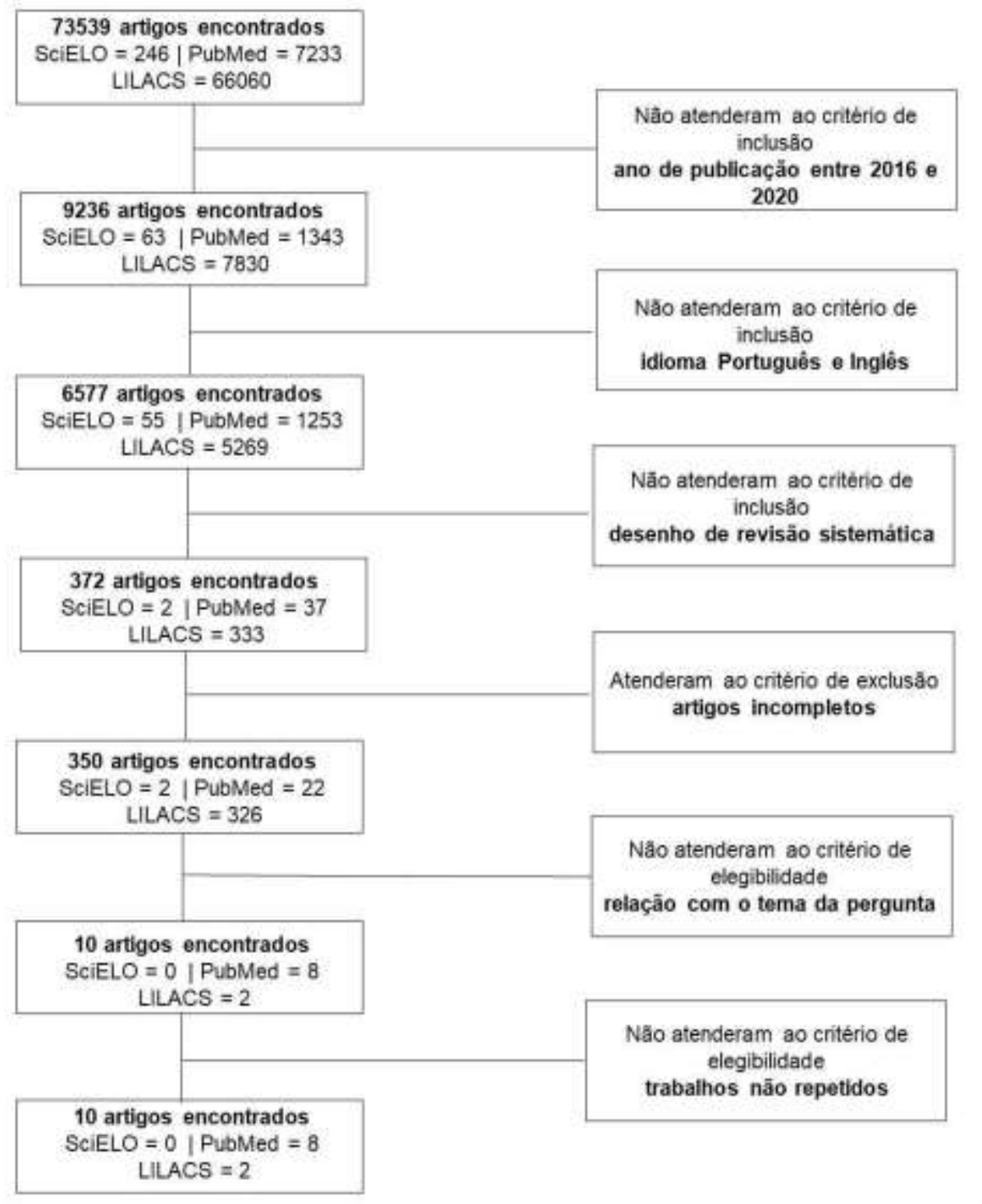

Fonte: Autores (2020). 
Quadro 1. Descrição sintetizada e níveis de evidência, segundo CASP adaptado, de cada estudo que compôs a revisão. Recife-

PE, 2020.

\begin{tabular}{|c|c|c|c|c|c|}
\hline Autor, ano & $\begin{array}{l}\text { País do } \\
\text { estudo }\end{array}$ & Amostra & Objetivo & Principais achados & $\begin{array}{c}\text { Evidências } \\
\text { (CASP } \\
\text { adaptado) }\end{array}$ \\
\hline $\begin{array}{l}\text { Alhomoud } \\
\text { et al., } 2017\end{array}$ & $\begin{array}{l}\text { Arábia } \\
\text { Saudita }\end{array}$ & 22 artigos & $\begin{array}{l}\text { Descobrir } \\
\text { porcentagem } \\
\text { próxima da realidade, } \\
\text { devido à falta de } \\
\text { pesquisas } \\
\text { sobre a automedicação } \\
\text { nas regiões do Oriente } \\
\text { Médio. }\end{array}$ & $\begin{array}{l}\text { Através da pesquisa, concluiu-se } \\
\text { que a maioria dos casos de } \\
\text { automedicação são realizados por } \\
\text { pacientes do sexo masculino; de } \\
\text { idade mais elevada; de baixa } \\
\text { renda, além do maior grau de } \\
\text { escolaridade. }\end{array}$ & A \\
\hline Ayalew, 2017 & Etiópia & $\begin{array}{c}21 \\
\text { artigos }\end{array}$ & $\begin{array}{l}\text { Prover uma análise da } \\
\text { literatura acerca da } \\
\text { prática de automedicação } \\
\text { entre os etíopes. }\end{array}$ & $\begin{array}{l}\text { A automedicação é prevalente na } \\
\text { Etiópia, variando de acordo com a } \\
\text { região e a população estudada. } \\
\text { Algumas ações de automedicação } \\
\text { são nocivas e necessitam de } \\
\text { intervenção urgentemente, como } \\
\text { dar-se maior ênfase à } \\
\text { normatização da venda de drogas. }\end{array}$ & A \\
\hline $\begin{array}{l}\text { Galvan et } \\
\text { al., } 2016\end{array}$ & Brasil & $\begin{array}{c}19 \\
\text { artigos }\end{array}$ & $\begin{array}{ll}\text { O estudo teve como } \\
\text { objetivo identificar as } \\
\text { evidências disponíveis } & \text { na } \\
\text { literatura acerca } & \text { da } \\
\text { automedicação entre os } & \text { os } \\
\text { profissionais de saúde. } & \end{array}$ & $\begin{array}{l}\text { A expectativas de solução } \\
\text { imediata de dores e sofrimentos, } \\
\text { bem como por terem acesso } \\
\text { facilitado aos medicamentos, os } \\
\text { profissionais de saúde buscam } \\
\text { alento na automedicação, sendo a } \\
\text { área médica mais jovem a que } \\
\text { mais usufrui de tal ação. Dessa } \\
\text { forma, a causa principal para o } \\
\text { usufruto da automedicação é a } \\
\text { cefaleia, que pode estar associada } \\
\text { ao estresse laboral. Os } \\
\text { medicamentos mais utilizados na } \\
\text { automedicação foram os } \\
\text { analgésicos, seguidos dos anti- } \\
\text { inflamatórios e antibióticos. }\end{array}$ & A \\
\hline $\begin{array}{l}\text { Khalifeh et al., } \\
\qquad 2017\end{array}$ & França & $\begin{array}{c}72 \\
\text { artigos }\end{array}$ & $\begin{array}{l}\text { O objetivo deste artigo, } \\
\text { portanto, é realizar uma } \\
\text { abrangente revisão para } \\
\text { identificar os diferentes } \\
\text { tipos de medicamentos } \\
\text { que podem ser facilmente } \\
\text { comprados } \\
\text { automedicação como } \\
\text { Oriente no } \\
\text { reconhecidos como mal } \\
\text { utilizados. }\end{array}$ & $\begin{array}{l}\text { A automedicação estava } \\
\text { significativamente associada com } \\
\text { a idade jovem, gênero masculino, } \\
\text { nível educacional e situação } \\
\text { socioeconômica, além do mau } \\
\text { estado de saúde. As pessoas } \\
\text { tendem a selecionar } \\
\text { medicamentos com base nos } \\
\text { conselhos recebidos do } \\
\text { farmacêutico comunitário, bem } \\
\text { como pais e amigos. A } \\
\text { automedicação é comum pela } \\
\text { experiência em usos anteriores em } \\
\text { doenças ou sintomas parecidos. } \\
\text { Analgésicos, anestésicos tópicos, } \\
\text { corticoides tópicos, produtos para } \\
\text { tosse e resfriado, antibiótico e } \\
\text { antimalária, foram as os } \\
\text { medicamentos mais usados. }\end{array}$ & A \\
\hline
\end{tabular}




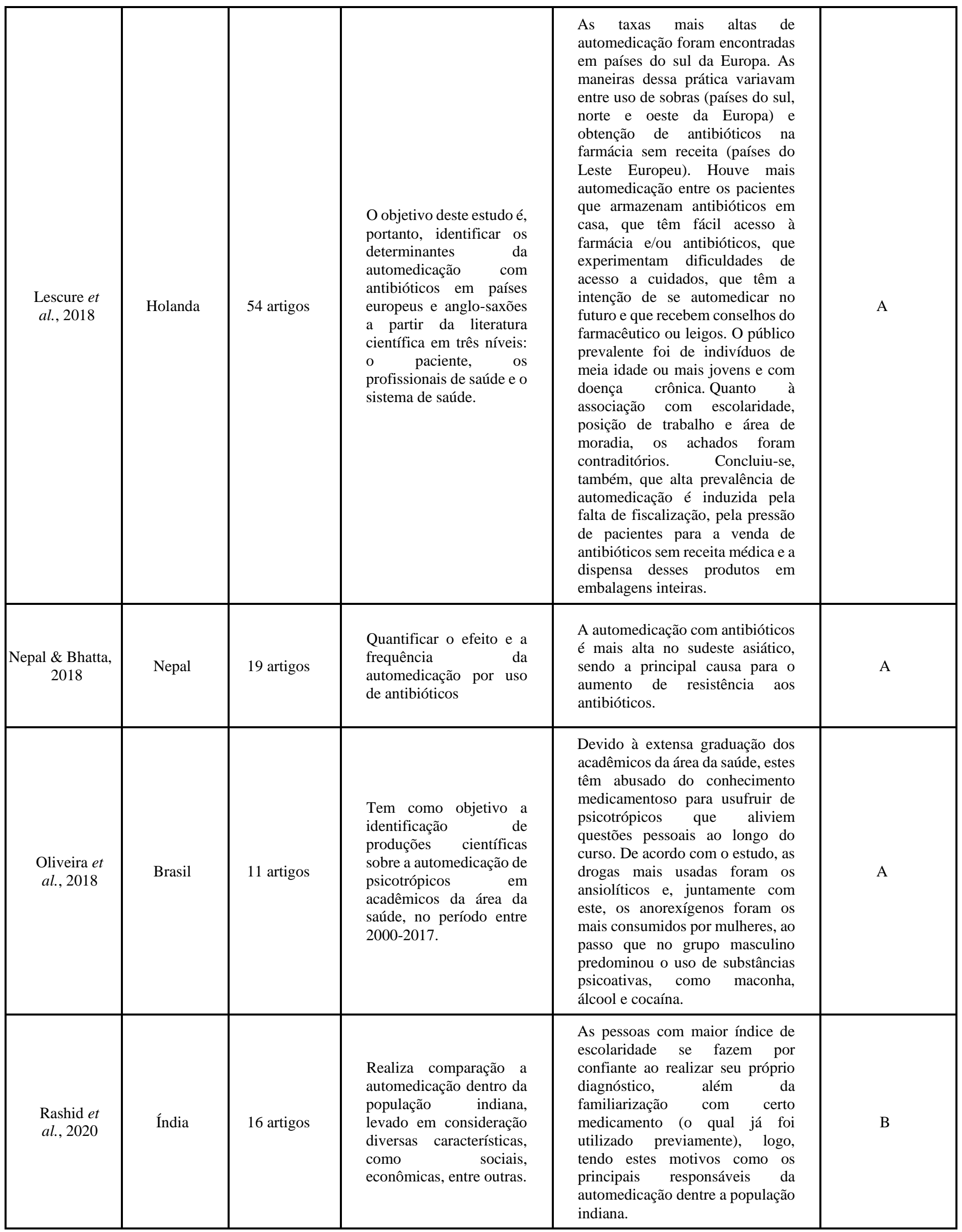




\begin{tabular}{|c|c|c|c|c|c|}
\hline $\begin{array}{c}\text { Sisay et al., } \\
2018\end{array}$ & Etiópia & $\begin{array}{c}27 \\
\text { estudos }\end{array}$ & $\begin{array}{l}\begin{array}{l}\text { Realizar uma } \\
\text { sistemática evisão } \\
\text { análise de }\end{array} \\
\begin{array}{r}\text { meta- } \\
\text { obstudos }\end{array} \\
\text { perfil epidemioniológico da } \\
\text { automedicação } \\
\text { etiópia. }\end{array}$ & $\begin{array}{l}\text { Chegando a resultados } \\
\text { secundários, o agrupado de } \\
\text { estimativas revelaram que os seis } \\
\text { motivos principais fornecidos por } \\
\text { clientes automedicados em ordem } \\
\text { decrescente eram exposição } \\
\text { anterior / familiaridade com os } \\
\text { tratamentos }(31,1 \%) \text {, } \\
\text { acessibilidade de automedicação } \\
(26,6 \%) \text {, economia de tempo } \\
(19,4 \%), \text { adequação para } \\
\text { atendimento de emergência } \\
(16,6 \%) \text { e menor expectativa de } \\
\text { unidades de saúde (13,6\%). Em } \\
\text { concordância com este estudo, um } \\
\text { estudo feito no Brasil indicou que } \\
\text { experiência anterior e/ou } \\
\text { familiaridade com a medicação foi } \\
\text { o principal motivo para } \\
\text { automedicação. }\end{array}$ & A \\
\hline $\begin{array}{l}\text { Xu et al., } \\
2019\end{array}$ & China & 49 artigos & $\begin{array}{l}\text { Comparação } \\
\text { porcentagem } \\
\text { automedicação por meio } \\
\text { de estudantes de diversos } \\
\text { países. }\end{array}$ & $\begin{array}{l}\text { Estudantes formados com PhD } \\
\text { possuem uma taxa de } \\
\text { automedicação de antibióticos } \\
\text { absurda, chegando a } 86,6 \% \text { dos } \\
\text { entrevistados. Além disso, quanto } \\
\text { mais avançavam nos períodos, } \\
\text { mais se automedicavam. } \\
\text { A Taxa de sexo e idades foram } \\
\text { irrelevantes em relação à } \\
\text { comparativa de dados. }\end{array}$ & A \\
\hline
\end{tabular}

Fonte: Autores (2020).

No que tange à automedicação, vê-se que os índices são maiores em indivíduos com maior escolaridade, principalmente entre os estudantes da área da saúde (Ayalew, 2017; Galvan et al., 2016; Oliveira et al., 2018; Rashid et al., 2019; Xu et al., 2019). Esse fator é observado por causa do conhecimento mais aprofundado sobre a doença e o medicamento para o seu tratamento.

Além disso, evidenciou-se que a automedicação é mais prevalente, em relação à idade, entre indivíduos mais jovens (Alhomoud et al., 2017; Galvan et al, 2016; Khalifeh et al., 2017). Isso indica que com o avançar da idade, as pessoas se tornam mais preocupadas com os potenciais transtornos que o uso de medicamento indiscriminado pode trazer para a saúde, seja por aconselhamento médico ou pelo próprio processo de reflexão individual. Entretanto, a prevalência entre as gestantes foi de grande destaque, em aproximadamente 22,9\% (Khalifeh et al., 2017; Sisay et al., 2018), evidenciando um potencial risco de saúde para a gestante e para o feto, devendo, assim, somente ser feita a medicação com orientação profissional.

Em relação aos fatores que convergem para o motivo da automedicação, tem-se, com maior prevalência, a experiência anterior no tratamento de sintomas parecidos, bem como a percepção de não haver gravidade e não precisar de atendimento médico (Ayalew, 2017; Nepal \& Bhatta, 2018; Xu et al., 2019); e isso tem grande relação com os principais sintomas que acometem a população, os quais são dor de cabeça, afecções do trato gastrointestinal e do sistema respiratório (Ayalew, 2017; Sisay et al., 2018).

As relações da comunidade também interferem e influenciam no uso indiscriminado de medicamentos. Isso porque, em estudos, observa-se que a obtenção desses medicamentos é feita em farmácias, principalmente por orientação de farmacêuticos, além dos adquiridos por amigos e familiares ou pelo uso das sobras de prescrições anteriores (Ayalew, 2017; Khalifeh et al., 2017; Nepal \& Bhatta, 2018; Rashid et al., 2020). É importante ressaltar que o risco de acontecer reações adversas por causa do uso de medicamentos sem aconselhamento e prescrição de um profissional adequado, com conhecimento na área, é 
extremamente potencializado.

As principais classes de medicamentos usados foram os analgésicos, anti-inflamatórios, antibióticos e fármacos com ação no sistema gastrointestinal (Galvan et al., 2016; Sisay et al., 2018). Isso ocorre porque são tratamentos de doenças comuns, conhecidas e que foram tratadas anteriormente com fármacos iguais ou similares. Além disso, a falta de tempo para consulta médica condiciona a automedicação para solução dessas doenças conhecidas (Ayalew, 2017).

No entanto, percebe-se que o uso indiscriminado de medicação sem prescrição prévia é uma preocupação em diversos países e, em especial, no tocante aos antibióticos, face ao aumento da resistência bacteriana a esse grupo de medicamentos (Nepal \& Bhatta, 2018). Mesmo a venda de antibióticos sendo feita somente com prescrição médica na maioria dos países, a venda livre ainda ocorre globalmente (Ayalew, 2017). Além disso, é fundamental ressaltar que grande parte dos estudos reforça a necessidade de normas legais que imponham maior controle às vendas das drogas em farmácias, bem como reafirmar o dever do poder público de dar maior visibilidade à temática, por meio de políticas educativas, com a finalidade de conscientizar a população como um todo acerca do uso de medicamentos sem a devida prescrição.

Com base no exposto, pode ser observado que há uma considerável amostra de artigos que, diga-se de passagem, apresenta uma diversidade cultural enorme. Destarte, a preocupação com a automedicação é observada em diversos países por grande parte do mundo, ainda que cada estudo apresente uma ótica particular face às subjetividades dos pesquisadores frente ao tema, mas também devido aos diferentes contextos que perpassam os determinantes sociais de cada local e cultura.

Em contrapartida, é notório que, devido à insuficiência de artigos relacionados à temática, o estudo permanece limitado, visto que há lacunas na literatura em função das baixas evidências, diversidade metodológica e restrição das bases de busca. Ou seja, ainda que este trabalho seja capaz de abarcar uma enorme gama de culturas, ainda não é possível atingir completamente todos os objetivos elencados anteriormente. Dessa forma, um fenômeno prevalente como a automedicação torna-se banalizado, com necessidade de obtenção de novos estudos que abranjam a compreensão dos aspectos laborais relacionados à prática mencionada, bem como seus efeitos e suas demandas de acordo com a especificidade de cada localidade.

\section{Conclusão}

Neste trabalho, foi possível realizar algumas associações entre a automedicação e os efeitos causados por esta ação, principalmente no tocante ao uso indiscriminado de antibióticos por parte da população. Entretanto, deve-se destacar que alguns objetivos apenas foram atingidos parcialmente. Isso é, foi possível: conhecer fatores que condicionam ao ato de se automedicar e identificar efeitos causados pela automedicação, porém avaliar a frequência do uso de medicamentos sem prescrição e estimar a prevalência de acordo com sexo e idade se mostrou um desafio face à revisão integrativa não ser o tipo de estudo mais adequado para se apontar prevalência ou incidência, além de haver um baixo número de pesquisas sobre este assunto.

Todavia, a temática trabalhada se mostrou um fator de preocupação em variados países pelo mundo, por isso, sendo extremamente relevante a discussão acerca da automedicação. Assim, pode-se concluir que a automedicação é um fenômeno de interesse internacional, com possível nocividade e que deve ser compreendida, estudada e trabalhada de maneira profunda através de estudos transversais para se obter dados mais concretos acerca da prevalência.

Diante disso, por meio da experiência vivenciada no presente trabalho, percebe-se a necessidade de mais produções científicas que abordem essa temática, de modo que permitam uma melhor avaliação não só quantitativa, como também qualitativa referente ao perfil prevalente de indivíduos que se automedicam a partir de fatores como, por exemplo, sexo e faixa etária. Assim, será possível, uma discussão mais adequada à questão, bem como a elaboração de trabalhos futuros. 


\section{Referências}

Alhomoud F, Aljamea Z, Almahasnah R, Alkhalifah K, Basalelah L, Alhomoud FK. Self-medication and self-prescription with antibiotics in the Middle Eastdo they really happen? A systematic review of the prevalence, possible reasons, and outcomes. Int J Infect Dis. 2017 Apr; 57:3-12. doi: 10.1016/j.ijid.2017.01.014. Epub 2017 Jan 19. PMID: 28111172.

Arrais, P. S., Fernandes, M. E., Pizzol, T. D., Ramos, L. R., Mengue, S. S., Luiza, V. L., Tavares, N. U., Farias, M. R., Oliveira, M. A., \& Bertoldi, A. D. (2016). Prevalence of self-medication in Brazil and associated factors. Revista de saude publica, 50(suppl 2), 13s. https://doi.org/10.1590/S15188787.2016050006117

Associação Médica Brasileira. (2001). Automedicação. Revista da Associação Médica Brasileira, 47(4), 269-270.

Ayalew M. B. (2017). Self-medication practice in Ethiopia: a systematic review. Patient Preference and Adherence, 11, 401-413. https://doi.org/10.2147/PPA.S131496

Conselho Federal de Farmácia (2019). Quase metade dos brasileiros que usaram medicamentos nos últimos seis meses se automedicou até uma vez por mês.

Domingues, P. H. F., Galvão, T. F., Andrade, K. R. C., Sá, P. T. T., Silva, M. T., \& Pereira, M. G. (2015). Prevalência da automedicação na população adulta do Brasil: revisão sistemática. Revista de Saúde Pública, 49(36), 1-8. https://doi.org/10.1590/S0034-8910.2015049005709

Galato, D., Madalena, J., \& Pereira, G. B. (2012). Automedicação em estudantes universitários: a influência da área de formação. Ciência \& Saúde Coletiva, 17(12), 3323-3330.

Galvan, M. R., Pai, M. R., \& Echevarría-Guanilo, M. E. (2014). Automedicação entre profissionais da saúde. http://www.dx.doi.org/10.5935/1415 2762.20160029

Instituto de Ciência, Tecnologia e Qualidade (2018). Pesquisa-Automedicação no Brasil.

Khalifeh, M. M., Moore, N. D., \& Salameh, P. R. (2017). Self-medication misuse in the Middle East: a systematic literature review. Pharmacology research \& perspectives, 5(4), $\mathrm{e} 00323$

Mendes, C. M. M. (2010). Perfil de automedicação em duas populações do município de Teresina [Dissertação de Mestrado, Universidade Federal do Ceará]. Repositório Institucional UFC. http://www.repositorio.ufc.br/handle/riufc/2268

Ministério da Saúde (2012). Dicas em Saúde: Automedicação. Biblioteca Virtual em Saúde.

Nepal, G., \& Bhatta, S. (2018). Self-medication with antibiotics in WHO Southeast Asian Region: a systematic review. Cureus, 10(4), 1-17. https://doi.org/10.7759/cureus.2428

Ogawa A. I., Kurachi G., Hata H. T., Abreu K. R. S., Lourenço L., Santos L. L., Matsumura M., Specian M. R., Elias T. V., Ivama A. M., Melchior S. C., Soares D. A., \& Turini B. (2001). Estudo comparativo sobre automedicação em estudantes do segundo ano de enfermagem e medicina e moradores do bairro Vila Nova. Espaço Saúde, 3(2), 13.

Oliveira, M. M., Corage, L. N., Oliveira, B. P., \& Silva, L. G. (2018). Automedicação de psicotrópicos em acadêmicos: uma revisão da literatura brasileira entre 2000 a 2017. Saúde e Pesquisa, 11(3), 623-630. https://doi.org/10.17765/1983-1870.2018v11n3p623-630

Organização Mundial da Saúde. (2004). How to investigate the use of medicines by consumers.

Paulo, L. G. \& Zanine A. C. (1988). Automedicação no Brasil. Revista da Associação Médica Brasileira, 34(2), 69-75.

Rashid, M., Chhabra, M., Kashyap, A., Undela, K., \& Gudi, S. K. (2020). Prevalence and Predictors of Self-Medication Practices in India: A Systematic Literature Review and Meta-Analysis. Current Clinical Pharmacology, 15(2), 90-101. https://doi.org/10.2174/1574884714666191122103953

Roman, A. R. \& Friedlander, M. R. (1998). Revisão integrativa de pesquisa aplicada à enfermagem. Revista Cogitare Enfermagem, 3(2), 109-112.

Sisay, M., Mengistu, G., \& Edessa, D. (2018). Epidemiology of self-medication in Ethiopia: a systematic review and meta-analysis of observational studies. BMC Pharmacology and Toxicology, 19(56), 1-12. https://doi.org/10.1186/s40360-018-0248-8

Xu, R., Mu, T., Wang, G., Shi, J., Wang, X., \& Ni, X. (2019). Self-Medication with Antibiotics among University Students in LMIC: A systematic review and meta-analysis. The Journal of Infection in Developing Countries, 13(8), 678-689. https://doi.org/10.3855/jidc.11359 